\title{
A logical formalization of integrity policies for database management systems
}

\author{
Frédéric Cuppens and Claire Saurel \\ ONERA-CERT \\ 2 Av. E. Belin, 31055, Toulouse Cedex, France. \\ Fax: +3362252593. \\ email: $\{$ cuppens, saurel\}otls-cs.cert.fr
}

\begin{abstract}
.
This paper presents a model to specify integrity policies for database management systems. This model makes it possible to (1) assign an integrity level to each user - this integrity level depends on the data this agent is authorized to update, (2) define updating permissions and prohibitions associated with each user -in particular we show that permission and prohibition to update may be independent from the user's integrity level, (3) define a policy to manage how integrity evolves in time. Our model is compared with classical approach, such as Biba and Clark-Wilson. In particular, we do not follow Biba: in our model, a subject may be authorized to update data even if its integrity level is not higher than or equal to the integrity level of the data.
\end{abstract}

Keyword Codes: D.4.6; F.4.1; H.2.0

Keywords: Security and Protection; Database Management, General; Mathematical Logic

\section{Introduction}

With confidentiality and availability, integrity is one of the three wellknown objectives of security which must be taken into account in information systems such as database management systems. Nevertheless few works have addressed this property, compared to the property of confidentiality. One famous integrity model is Biba's one (Biba, 1976). This early model is directly derived from the Bell and LaPadula model (Bell and LaPadula, 1975): every object and subject is associated with an integrity level. The set of integrity levels is associated with a partial order relation. Biba's model can then be summerized by the two following constraints: (1) a subject is allowed to write an object only if the integrity level of this subject is higher than or equal to the integrity level of this object, (2) a subject is allowed to read an object 
only if the integrity level of this subject is lower than or equal to the integrity level of this object.

In the context of databases, Biba's model does not always fit with real requirements. In particular, a subject may need to update data even if the subject's integrity level is not higher than or equal to the integrity level of the data; moreover, in many applications, its updating rights may not directly depend on its integrity level.

In this paper, we first claim that there is a need for a temporal database to overcome these difficulties. Our objective is then to suggest a formal model of an integrity policy to manage updating in a temporal database. For any given database, we want our model to be able to:

- assign an integrity level to each user, depending on the data it is authorized to modify/update,

- define updating permissions and prohibitions for each user,

- define a policy to manage how integrity evolves in time.

The remainder of this paper is organized as follows. Section 2 will introduce our main motivations for this work. We show within two simple examples that Biba's model is clearly insufficient in concrete applications, and that there is a real need for temporal databases if one wants to manage integrity of data. From these examples, we will derive the main ideas which support our model. Section 3 explains our approach and justifies the different steps we use to achieve our objectives. In the fourth section the concept of multi-reliable database is defined to address the property of integrity. Roughly speaking, this concept may be viewed as similar to the well-known concept of multilevel database which has been suggested to manage a multilevel confidentiality policy. Section 5 presents a logical formalization for the notion of reliability of an agent which has to update data in a multi-reliable database; for a given agent, reliability depends on the nature of the data which must be updated. Moreover, the integrity of a given data is not static but may evolve in time. For this purpose, section 6 introduces a predicate called revise-integrity in order to formalize revision of data integrity in time. Section 7 then shows how we can model an integrity policy which regulates data updates. In section 8 , this model is applied to formalize the two initial examples of section 2 . Finally section 9 concludes this paper by comparing this work with other former works, and investigating several issues. 


\begin{tabular}{|l|l|l|l|}
\hline N\# & POS & Validity & Integrity \\
\hline sh2 & pos 1 & {$[12: 00,12: 01[$} & HI \\
sh2 & pos 2 & {$[12: 01,12: 02[$} & LI \\
sh2 & $p o s 3$ & {$[12: 02,12: 03[$} & LI \\
$\ldots$ & $\ldots$ & $\ldots .$. & $\ldots$ \\
sh2 & $p o s 60$ & {$[12: 59,13: 00[$} & LI \\
sh2 & pos61 & {$[13: 00,13: 01[$} & HI \\
sh2 & pos62 & {$[13: 01$, now $]$} & LI \\
\hline
\end{tabular}

Figure 1. Instance of the relation Position

\section{Motivations}

This section presents two examples to show some insufficiency in Biba's model, and some desirable properties for the concept of integrity.

\subsection{EXAMPLE 1}

Let us consider two ships sh1 and sh2, with sh1 continuously trying to know sh2's position. sh1 receives information from two sources: a satellite $s$ which provides highly reliable data on each hour, and a lower reliable radar $r$ on board of $s h 1$, which provides information each minute. In a situation of emergency, the estimation of $s h 2$ 's position must be sufficiently precise, so that it cannot be based only upon the data coming from the satellite each hour; therefore, sh1 must take both data provided by $s$ and $r$ into account.

Using a temporal database, we represent the position of sh2 with a relation Position: its attributes respectively represent the identifier of a ship, the value of its position, the time during which a given position is supposed to be valid, and the integrity level of this position. Figure 1 gives a set of instances of this relation.

We can notice that:

- Here, as in most temporal databases, sh2's position is assumed not to change as long as this position is not updated (each minute, in our example); it is a default assumption. For this purpose, a special value now is used, with the meaning "true until changed". 
- Despite Biba's model, data provided by $r$ updates data provided by $s$, although $r$ 's integrity level LI (Low Integrity) is lower than the integrity level HI (High Integrity) of data $s$ provides. As a matter of fact, in this application, it would not be sufficient to only deal with data provided by $s$. This example shows that in some applications, there is a real need for authorizing updates even though the integrity level of the subject performing the update is lower than the integrity level of the updated object.

- We need to keep track of high level data coming from $s$. Such records may be useful to compute trajectory of sh2 or to check for consistency of data coming from $s$ and $r$. This goal may be accomplished by using a temporal database.

- When data are provided by $s$ and $r$ at the same time, the database only keeps data coming from $s$ because $s$ is more reliable. In this case, data coming from $s$ has priority over data coming from $r$.

- Now let us assume that the satellite $s$ is the only source of information providing $s h 1$ with the position of $s h 2$. Most of classical temporal databases would consider that the position of $s h 2$ remains unchanged until $s$ provides new data (event which only occurs each hour). However, since sh2 moves in time, it would not be safe to consider that the position provided by $s$ is always highly reliable during one hour. Figure 2 provides a more realistic representation of the integrity of data provided by $s$.

In this figure, the integrity level of the fact "sh2's position is pos" becomes lower and lower in time, with a degradation speed depending on some parameters such as for instance sh2's supposed speed. Of course the position is highly reliable at the beginning of each hour, that is when the position is updated by the satellite. Then, the integrity level of sh2's position changes in time. In figure 2, it is assumed that this integrity decreases at medium (MI) after one minute, and then at low (LI) after three minutes. 


\begin{tabular}{|l|l|l|l|}
\hline N\# & POS & Validity & Integrity \\
\hline sh2 & pos 1 & {$[12: 00,12: 01[$} & HI \\
sh2 & pos 1 & {$[12: 01,12: 03[$} & MI \\
sh2 & pos 1 & {$[12: 03,13: 00[$} & LI \\
sh2 & pos 2 & {$[13: 00,13: 01[$} & HI \\
sh2 & pos 2 & {$[13: 01,13: 03[$} & MI \\
sh2 & pos 2 & {$[13: 03$, now $]$} & LI \\
\hline
\end{tabular}

Figure 2. Integrity decreasing of the position in time

\subsection{EXAMPLE 2}

Let us assume we are in a company where a secretary $s 1$ has to manage and update employees' salaries. $s 1$ has a high integrity level for this task. Another secretary $s 2$ has to manage employees' vacation. $s 2$ has a high integrity level for this task.

Now, let us assume that during $s 1$ 's vacation, a temporary secretary $s 3$ is hired and is authorized to update the salary data file, with a medium level of integrity. On the other hand, $s 3$ has also to replace $s 2$ during $s 2$ 's vacation, so that $s 3$ is also authorized to update the vacation data file, with a high integrity level.

In this example, we can see that:

- The integrity level of an agent considered as a source of information may depend on the nature of data this agent updates;

- The update rights of an agent do not depend on this agent's integrity level: despite $s 2$ could be very reliable to update the salary data file, it is not authorized to do so; however, even though $s 3$ 's integrity level is medium, it is allowed to update this file during $s 1$ 's vacation, and is not allowed to do so at any other time.

- The agent's rights for a task may depend on time; $s 3$ is allowed to update the salary file during $s 1$ 's vacation only. 


\subsection{REQUIREMENTS SUPPORTING OUR APPROACH}

From the two above examples, we claim that:

1. The agent's reliability may depend on the data it updates.

2. The agent's reliability may change in time.

3. The agent's updating rights may not depend on its reliability.

4. Sometimes, an agent must be able to update a data even if its reliability level is lower than the reliability level of the data which is updated (despite Biba's model).

5. The agent's updating rights may change in time.

6. It is necessary to introduce a temporal representation of data to prevent a data with a low integrity level from erasing a data with a higher integrity level.

7. Without any update, a data integrity level may decrease in time, according to some characteristics of a given application.

\section{Main steps of our approach}

We first define the concept of multi-reliable database. It is similar to the concept of multilevel database to deal with the property of confidentiality: data have labels which denote their level of integrity. We shall consider that a multi-reliable database is a set of beliefs and we shall define what does it means for a multi-reliable database to believe that a given data is associated with a given level of integrity.

As for confidentiality, we also assign an integrity (or reliability) level to each agent which is responsible for updating the database, i.e. which can be considered as a source of data: in our model, the integrity level of an agent depends on the data it updates. We also use temporal notions to model the fact that the integrity level of an agent may change in time.

We also have to express that a data integrity level may change in time. For this purpose, we shall introduce an event revise-integrity: the occurrence of this event automatically updates the level of integrity of a 
given data. Then it becomes possible to define, according to insertions, deletions or revisions of data, when a multi-reliable database believes that a given data has a given level of integrity.

Then we suggest a formalization for the concept of integrity policy. We claim that an integrity policy has three components:

1. The first one is the assignment of reliability levels to agents, depending on the data they may update.

2. The second one is a regulation of rights and prohibitions of agents towards insertions and deletions in the database.

3. The last one is a set of rules specifying how the integrity level of data has to be revised.

In this paper, we do not deal with integrity constraints, i.e. rules which must be satisfied by any state of the database. This does not mean that we consider that integrity constraints are not included in the concept of integrity. In particular, we guess there is a clear connection between integrity constraints and the notion of well-formed transaction suggested in the Clark-Wilson model (Clark and Wilson, 1987; Clark and Wilson, 1989). Therefore, despite we do not take them into account in our model for an integrity policy, such rules have to be specified and enforced by the multi-reliable database.

\section{Formalization of a multi-reliable database}

Let $I L$ be a set of integrity levels associated with a partial order relation denoted $\unlhd$ (that gives a lattice structure). If $n_{1}$ and $n_{2}$ are two integrity levels of $I L, n_{1} \unlhd n_{2}$ means that $n_{1}$ is lower than or equal to $n_{2}$. For instance, if $\mathrm{HI}$ (high integrity), MI (medium integrity) and LI (low integrity) are in $I L$, then we have LI $\unlhd$ MI $\unlhd$ HI. We also introduce an unary predicate Level with formula of the form Level $(n)$ to be read " $n$ is an integrity level".

We denote $\triangleleft$ the strict order relation derived from $\unlhd$; we have the following axiomatics for $\triangleleft$ :

$$
\text { - } \forall n_{1}, \forall n_{2}, n_{1} \triangleleft n_{2} \leftrightarrow n_{1} \unlhd n_{2} \wedge \neg\left(n_{1}=n_{2}\right)
$$


Now we consider that a multi-reliable database is composed of several single-level databases; each of them is assigned with an integrity level $n$ and is the set of all the data which are explicitly assigned with an integrity level higher than or equal to $n$. We also consider that each single-level database represents a set of beliefs. Therefore, to represent the content of each single-level database, we introduce a modality believe with formula of the form believe $(n, p)$ to be read : "the database with integrity level $n$ believes that the information $p$ is valid".

The axiomatics associated with believe $(n, p)$ is a $\mathrm{KD}$ logic (Chellas, 1988):

- K: $($ believe $(n, p) \wedge$ believe $(n, p \rightarrow q)) \rightarrow \operatorname{believe}(n, q)$

(closure of believeness within the database of integrity level $n$ )

- D: believe $(n, p) \rightarrow \neg$ believe $(n, \neg p)$

(consistency of the database associated with the integrity level $n$ )

- $\quad \mathrm{N}$ : If $p$ is a theorem then $\forall n \in I L$, believe $(n, p)$ is also a theorem.

(the database associated with level $n$ believes all theorems)

We also have the following additional axiom:

- $\forall n, \forall n^{\prime},\left(\operatorname{believe}(n, p) \wedge n^{\prime} \unlhd n\right) \rightarrow \operatorname{believe}\left(n^{\prime}, p\right)$

(if the database of level $n$ believes $p$ then all databases of level $n^{\prime}$ lower than $n$ also believe $p$ )

Then we assign an integrity label to every information derived from the multi-reliable database. For this purpose, we introduce a metapredicate safety_level, where safety_level $(p, n)$ is to be read: " the integrity level of information $p$ is $n$ ". The axiomatics associated with safety_level $(p, n)$ is:

- $\forall n \in I L$, safety_level $(p, n) \leftrightarrow$

$$
\text { (believe } \left.(n, p) \wedge \forall n^{\prime}, n \triangleleft n^{\prime} \rightarrow \neg \text { believe }\left(n^{\prime}, p\right)\right)
$$

(the integrity level of $p$ is $n$ iff the database of level $n$ believes $p$ and all databases with level $n^{\prime}$ higher than $n$ do not believe $p$ ).

Finally, we check that the database at level $n$ does not believe $p$ by testing omission of explicit believe of $p$, that is: 
- If $\nvdash$ believe $(n, p)$ then $\vdash \neg$ believe $(n, p)$

In the remainder of this paper, we shall actually consider that our multi-reliable database contains temporal data. For this purpose and following Sripada (Sripada, 1993), we shall introduce two additional modalities hold_at and hold with formula of the form hold_at $(p, T)$ to be read " $p$ is valid at time $T$ " and formula of the form $\operatorname{hold}\left(p,\left[T_{1}, T_{2}\right]\right)$ to be read " $p$ is valid on the interval of time $\left[T_{1}, T_{2}\right]$ ". For instance, formula:

- hold_at(believe(HI, hold_at(Position(sh2, pos1), 12:00)), $13: 00)$

is to be read: "at time 13:00, the database associated with the integrity level $H I$ believes that the position of $\operatorname{sh} 2$ is pos 1 at time 12:00". In this formula, time 12:00 is generally called a valid time (or an historic time) and time 13:00 is called a transaction time (or a belief time) (Snodgrass and Ahn, 1985).

Due to space limitation, we do not develop the axiomatics associated with modalities hold_at and hold, but see (Sripada, 1993) and (Cuppens and Saurel, 1998) for a more detailed presentation. In the following, we shall also use a special value now with formula of the form hold_at ( $p$, now) to be read " $p$ is valid until changed" (see (Clifford et al., 1997) and (Cuppens and Saurel, 1998) for a detailed presentation of the semantics and axiomatics associated with now).

The multi-reliable database we consider contains temporal data having the form hold(believe $\left.\left(n, \operatorname{hold}\left(p, I_{1}\right)\right), I_{2}\right)$ where $n$ is an integrity level, $p$ is an atomic formula and $I_{1}$ and $I_{2}$ are two time intervals. To represent modification performed in a multi-reliable database, we shall consider the following two meta-predicates:

- insert. Formulae of the form insert $(a, p, I)$ are to be read "agent $a$ has inserted in the multi-reliable database the fact that $p$ is valid on the interval I".

- delete. Formulae of the form delete $(a, p, I)$ are to be read "agent $a$ has deleted from the multi-reliable database the fact that $p$ is valid on the interval I".

We could also define a third operation update. However, this last operation may be viewed as a combination of delete and insert. Therefore, it is not necessary to include this operation in our model. 


\section{Reliability of an agent}

Here we formalize the notion of reliability of agents who update the multi-reliable database. As we want the reliability of an agent to depend on the updated data, we introduce a meta-predicate safer_than where formula of the form safer_than $(a, p, n)$ is to be read ; "the integrity level of data $p$ is higher than or equal to $n$ when it is the agent $a$ who inserts $p$ in the database".

Actually, we consider that agents always insert, in the database, data of the form hold $\left(p,\left[T_{1}, T_{2}\right]\right)$. Therefore, we suggest the following axiom to express what is the reliability of an agent when this agent inserts some data in the multi-reliable database:

$$
\begin{aligned}
& \text { - } \forall a, \forall T_{1}, \forall T_{2}, \forall n, \text { safer_than }\left(a, \operatorname{hold}\left(p,\left[T_{1}, T_{2}\right]\right), n\right) \leftrightarrow \\
& \text { believe }\left(n, \operatorname{insert}\left(a, p,\left[T_{1}, T_{2}\right]\right) \rightarrow \operatorname{hold}\left(p,\left[T_{1}, T_{2}\right]\right)\right)
\end{aligned}
$$

i.e. safer_than $\left(a, \operatorname{hold}\left(p,\left[T_{1}, T_{2}\right]\right), n\right)$ is true iff the database associated with the integrity level $n$ believes that if the agent $a$ inserts $\operatorname{hold}\left(p,\left[T_{1}, T_{2}\right]\right)$ in the database, then $p$ is actually valid on the interval $\left[T_{1}, T_{2}\right]$. In other words, safer_than $\left(a, \operatorname{hold}\left(p,\left[T_{1}, T_{2}\right]\right), n\right)$ is true iff the database of level $n$ believes what agent $a$ tells about $\operatorname{hold}\left(p,\left[T_{1}, T_{2}\right]\right)$.

In the following, we shall also use the predicate insert ${ }^{\prime}$ defined as follows:

- $\operatorname{insert}^{\prime}(p, I, n)$ is to be read "data $\operatorname{hold}(p, I)$ is inserted in the database with an integrity level greater than $n$ ". We have:

$$
\begin{aligned}
& \forall T_{1}, \forall T_{2}, \forall n, \operatorname{insert}^{\prime}\left(p,\left[T_{1}, T_{2}\right], n\right) \leftrightarrow \\
& \quad \exists a, \operatorname{insert}\left(a, p,\left[T_{1}, T_{2}\right]\right) \wedge \operatorname{safer\_ than}\left(a, \operatorname{hold}\left(p,\left[T_{1}, T_{2}\right]\right), n\right)
\end{aligned}
$$

\section{Evolution of a data integrity level in time}

To represent the fact that the integrity level of data may change in time, we introduce a predicate revise_integrity with formula of the form revise_integrity $(p, I, n)$ to be read: "the integrity level of data $\operatorname{hold}(p, I)$ is revised at level $n$ ".

From predicates insert ${ }^{\prime}$, delete and revise_integrity, we define three other predicates insertion, deletion and revision_integrity as follows: 
- Intuitively, insertion $(p, T, n)$ means "data hold_at $(p, T)$ is inserted in the database associated with the integrity level $n$ ". If $T$ is a date (not the special value now), then there are two possible cases:

1. Data $\operatorname{hold}\left(p,\left[T_{1}, T_{2}\right]\right)$ is inserted in the database of integrity level $n$ and time $T$ is between $T_{1}$ and $T_{2}$.

2. Data hold ( $p,\left[T_{1}\right.$, now $\left.]\right)$ is inserted in the database of integrity level $n$, and $T$ is between $T_{1}$ and the date of reference. Roughly speaking, the date of reference represents the "present date"; it makes it possible to interpret the special value now (see (Cuppens and Saurel, 1998)).

Therefore, we have:

$$
\begin{array}{r}
\forall T \in \begin{array}{l}
\left.\forall \text { Date, }, \forall n, \text { insertion }_{(}, T, n\right) \leftrightarrow \\
\exists T_{1}, \exists T_{2},\left(\text { insert }^{\prime}\left(p,\left[T_{1}, T_{2}\right], n\right) \wedge T_{1} \preceq T \wedge T \preceq T_{2}\right) \\
\vee \quad\left(\text { insert }^{\prime}\left(p,\left[T_{1}, \text { now }\right], n\right) \wedge\right. \\
\left.\quad \text { Reference_date }\left(T_{2}\right) \wedge T_{1} \preceq T \wedge T \preceq T_{2}\right)
\end{array}
\end{array}
$$

If $T$ is equal to now, we have:

$\forall n$, insertion $(p$, now,$n) \leftrightarrow \exists T, \operatorname{insert}^{\prime}(p,[T$, now $], n)$

- deletion $(p, T)$ means "data hold_at $(p, T)$ is deleted from the multireliable database". Following the definition of insertion, if $T$ is a date, we have:

$$
\begin{aligned}
& \forall T \in \text { Date, deletion }(p, T) \leftrightarrow \\
& \begin{array}{l}
\exists T_{1}, \exists T_{2}, \exists a,\left(\text { delete }\left(a, p,\left[T_{1}, T_{2}\right]\right) \wedge T_{1} \preceq T \wedge T \preceq T_{2}\right) \\
\vee\left(\text { delete }\left(a, p,\left[T_{1}, \text { now }\right]\right) \wedge\right.
\end{array} \\
& \left.\quad \text { Reference_date }\left(T_{2}\right) \wedge T_{1} \preceq T \wedge T \preceq T_{2}\right)
\end{aligned}
$$

And if $T$ is equal to now:

$$
\operatorname{deletion}(p, \text { now }) \leftrightarrow \exists a, \exists T, \operatorname{delete}(a, p,[T, \text { now }])
$$

- revision_integrity $(p, T, n)$ means "the integrity level of data hold_at $(p, T)$ is revised at level $n$ ". Following the definition of insertion, if $T$ is a date, we have: 
$\forall T \in$ Date,$\forall n$, revision_integrity $(p, T, n) \leftrightarrow$

$$
\begin{gathered}
\exists T_{1}, \exists T_{2},\left(\text { revise_integrity }\left(p,\left[T_{1}, T_{2}\right], n\right) \wedge T_{1} \preceq T \wedge T \preceq T_{2}\right) \\
\vee\left(\text { revise_integrity }\left(p,\left[T_{1}, n o w\right], n\right) \wedge\right. \\
\left.\quad \text { Reference_date }\left(T_{2}\right) \wedge T_{1} \preceq T \wedge T \preceq T_{2}\right)
\end{gathered}
$$

And if $T$ is equal to now:

$\forall n$, revision_integrity $(p$, now,$n) \leftrightarrow$

$$
\exists T, \text { revise_integrity }(p,[T, \text { now }], n)
$$

Now, we introduce two additional predicates apparent_integrity and real_integrity. These two predicates are defined as follows:

- apparent_integrity $(p, T, n)$ means "the apparent integrity of data hold_at $(p, t)$ is higher than or equal to $n$ "; that is, at a previous date, the integrity of $h o l d \_a t(p, T)$ was higher than or equal to $n$, but it is possible that, since this date, data hold_at $(p, T)$ or its integrity level has been updated.

$\forall n$, apparent_integrity $(p, T, n) \leftrightarrow$

(insertion $(p, T, n)$

$\vee \exists n^{\prime}$, revision_integrity $\left.\left(p, T, n^{\prime}\right) \wedge n \unlhd n^{\prime}\right)$

- real_integrity $(p, T, n)$ means "the integrity of data hold_at $(p, T)$ is higher than or equal to $n$ ".

$\forall T, \forall n$, real integrity $(p, T, n) \leftrightarrow \exists T_{1}, \exists T_{2}$,

(Reference_date $\left(T_{1}\right) \wedge$ hold_at(apparent_integrity $\left.(p, T), T_{2}\right) \wedge T_{2} \preceq T_{1} \wedge$

$\neg \exists T_{3},\left(T_{2} \prec T_{3} \wedge T_{3} \preceq T_{1} \wedge\right.$ hold_at $\left(\right.$ deletion $\left.\left.(p, T), T_{3}\right)\right) \wedge$ $\neg \exists T_{3}, \exists n^{\prime},\left(T_{2} \prec T_{3} \wedge T_{3} \preceq T_{1}\right.$

$\wedge$ hold_at $\left(\right.$ revision_integrity $\left.\left.\left.\left(p, T, n^{\prime}\right), T_{3}\right) \wedge n^{\prime} \triangleleft n\right)\right)$

that is: the integrity of data hold_at $(p, T)$ is higher than or equal to $n$ if and only if:

1. Data hold_at $(p, T)$ had an integrity level higher than or equal to $n$ at a date $T_{2}$ which is before the reference date $T_{1}$.

2. Data hold_at $(p, T)$ was not deleted between $T_{2}$ (excluded) and $T_{1}$ (included). 
3. The integrity level of data hold_at $(p, T)$ was not updated at an integrity level $n^{\prime}$ strictly dominated by $n$ between $T_{2}$ (excluded) and $T_{1}$ (included).

We can now define in which situation the database associated with the integrity level $n$ believes that data hold_at $(p, T)$ is valid. For this purpose, we suggest the following axioms:

- $\forall T_{1}, \forall T_{2}, \forall T, \forall n \in I L,\left(\right.$ Reference_date $\left.\left(T_{2}\right) \wedge T_{1} \preceq T_{2}\right) \rightarrow$ hold_at(believe $(n$, hold_at $(p, T) \leftrightarrow$ real_integrity $\left.(p, T, n)), T_{1}\right)$ that is: if $T_{1}$ is a date before the reference date $T_{2}$, then the database at level $n$ believes at time $T_{1}$ that the fact $p$ (where $p$ represents any atomic fact the multi-reliable database may contain) is valid at time $T$ if and only if the real integrity level of data hold_at $(p, T)$ is higher than or equal to $n$.

- $\forall T_{1}, \forall T_{2}, \forall T, \forall n \in I L,\left(\right.$ Reference_date $\left.\left(T_{2}\right) \wedge T_{2} \prec T_{1}\right) \rightarrow$ hold_at $\left(\neg\right.$ believe $(n$, hold_at $\left.(p, T)), T_{1}\right)$

that is: if $T_{1}$ is a date strictly after the reference date $T_{2}$, then the database at level $n$ does not believe, at time $T_{1}$, that $p$ is valid at time $T$ (where $p$ represents any atomic fact the multi-reliable database may contain). This axiom corresponds to the assumption that a transaction time must always be before the reference date.

\section{Integrity policy}

We now want to formalize an integrity policy which regulates updates in a multi-reliable database. This is done in three steps:

1. Assignment of integrity levels to the users of the multi-reliable database. This is done by using the predicate $\operatorname{safer} \_\operatorname{than}(a, p, n)$ introduced in section 5 .

2. Specification of rights and prohibitions for agents who can insert or delete data in the database. For this purpose, we introduce two deontic modalities $\mathbf{P}$ (permission) and $\mathbf{I}$ (prohibition).

For instance, the formula 
$\mathbf{P}$ insert(Clementine, In_vacation(Jane), [1/11/97, 15/11/97])

is to be read" Clementine is permitted to insert in the database the fact: Jane is in vacation from November, 1st to November, 15th.

3. Definition of the policy which regulates the evolution of integrity in time. The objective is to give rules which specify how data integrity has to be revised. For this purpose, we introduce a third modality $\mathbf{O}$ where formula of the form $\mathbf{O}$ revise_integrity $\left(p,\left[T_{1}, T_{2}\right], n\right)$ is to be read "the integrity level of hold $\left(p,\left[T_{1}, T_{2}\right]\right)$ must be revised to the level $n$ ".

The axiomatics of deontic modalities $\mathbf{P}, \mathbf{I}$ and $\mathbf{O}$ is defined as follows:

- $\mathbf{O}$ (obligation) is associated with the axiomatics of SDL (Standard Deontic Logic) (Meyer and Wieringa, 1991), which actually corresponds to $\mathrm{KD}$ logics for $\mathbf{O}$ :

$\mathbf{K}:(\mathbf{O} p \wedge \mathbf{O}(p \rightarrow q)) \rightarrow \mathbf{O} q$

(if $p$ is obligatory and if $p \rightarrow q$ is obligatory then $q$ is obligatory).

$\mathrm{D}: \mathbf{O} p \rightarrow \neg \mathbf{O} \neg p$

(if $p$ is obligatory then $\neg p$ is not obligatory).

$\mathrm{N}$ : If $p$ is a theorem then $\mathbf{O} p$ is also a theorem.

(all theorems are obligatory).

- $\mathbf{I} p \stackrel{\text { def }}{=} \mathbf{O} \neg p$

( $p$ is forbidden iff the negation of $p$ is obligatory).

- $\mathbf{P} p \stackrel{\text { def }}{=} \neg \mathbf{I} p$

( $p$ is authorized iff $p$ is not forbidden).

Using the formalism we have just defined, we can now specify an integrity policy as follows:

1. Definition of predicate safer_than and,

2. Definition of conditions under which $\mathbf{P} \operatorname{insert}\left(a, p,\left[T_{1}, T_{2}\right]\right)$ and $\mathbf{P}$ delete $\left(a, p,\left[T_{1}, T_{2}\right]\right)$ hold and, 
3. Conditions under which $\mathbf{O}$ revise_integrity $\left(p,\left[T_{1}, T_{2}\right], n\right)$ holds.

Moreover we make the following closed-world assumption: if an agent $a$ is not explicitly authorized to insert or delete $\operatorname{hold}\left(p,\left[T_{1}, T_{2}\right]\right)$, then $a$ is forbidden to do so. This means that an agent must be explicitly authorized to perform any insertion or deletion in the database.

As mentioned before, we have also to deal with integrity constraints. Integrity constraints may be either state constraints (e.g, the position of a ship is unique at a given time), or transition constraints (e.g, in an hour, a ship cannot move more than 60 miles). In our approach, we assume that the state of the database reached when performing any insertion or deletion satisfies the set of integrity constraints: if it does not, the insertion or deletion is rejected.

The next section shows how to specify examples of integrity policies formalized within our language.

\section{Examples of formalized integrity policies}

\subsection{Example 1: Position of a Ship}

We use the following predicate symbols to formalize this first application.

- Ship. Ship $(x)$ means " $x$ is a ship".

- Val_Pos.Val_Pos $(y)$ means " $y$ is the value of a position".

- Position. Position $(x, y)$ means "the position of $x$ is $y$ ".

We then specify one typing constraint:

$$
\text { - } \forall x, \forall y, \operatorname{Position}(x, y) \rightarrow \operatorname{Ship}(x) \wedge V a l \_P o s(y)
$$

and one integrity constraint, claiming that the position of a ship is unique at any given time:

$$
\text { - } \forall x, \forall y, \forall y^{\prime}, \operatorname{Position}(x, y) \wedge \operatorname{Position}\left(x, y^{\prime}\right) \rightarrow y=y^{\prime}
$$

Let us assume we have two information sources, a satellite $s$ and a radar $r$, which is supposed to be jammed.

The first step in our approach consists in specifying their level of reliability as follows: 
- $\forall x, \forall y, \forall T, S h i p(x) \wedge V a l \_P o s(y) \wedge \operatorname{Date}(T)$

$\rightarrow$ safer_than $(s, \operatorname{hold}(\operatorname{Position}(x, y),[T$, now $]), H I)$

(the satellite is highly reliable when it tells about the position of a ship).

- $\forall x, \forall y, \forall T, S h i p(x) \wedge V a l \_P o s(y) \wedge \operatorname{Date}(T) \wedge \operatorname{Jammed}(r)$

$\rightarrow$ safer_than $(r$, hold(Position $(x, y),[T$, now $]), L I)$

(if the radar is jammed, then its integrity level is low when it tells about the position of a ship).

The second step consists in defining the update policy:

- $\forall x, \forall y, \forall T, \operatorname{Ship}(x) \wedge$ Val_Pos $(y) \wedge \operatorname{Date}(T)$

$\rightarrow \mathbf{P}$ insert $(s$, Position $(x, y),[T$, now $])$

the satellite is allowed to insert the position of any ship.

- $\forall x, \forall y, \forall T, \operatorname{Ship}(x) \wedge$ Val_Pos $(y) \wedge \operatorname{Date}(T)$

$\rightarrow \mathbf{P}$ insert $(r, \operatorname{Position}(x, y),[T$, now $])$

the radar is also allowed to insert the position of any ship.

- $\forall x, \forall y, \forall T, S h i p(x) \wedge V a l \_P o s(y) \wedge \operatorname{Date}(T)$

$\rightarrow \mathbf{P}$ delete $(s$, Position $(x, y),[T$, now $])$

the satellite is allowed to delete the position of any ship. So by combining both rights for insertion and deletion, the satellite is then allowed to update the position of any ship.

- For the radar, the situation is more complex. Since the satellite is more reliable than the radar, we do not want the radar to be allowed to update any data which has just been given by the satellite. However, we are interested in getting information from the radar when information coming from the satellite becomes obsolete. So the policy allows the radar to update (by two successive deletion and insertion operations) a position given by the satellite only after a given time, say after at least one minute. So we have:

$$
\begin{aligned}
& \forall x, \forall y, \forall T \text {, } \\
& \operatorname{Ship}(x) \wedge V a l \_P o s(y) \wedge \operatorname{Date}(T) \wedge \\
& \neg \exists T_{1}, \exists T_{2},\left(\text { hold_at }\left(\text { insert }\left(s, \text { Position }(x, y),\left[T_{1}, \text { now }\right]\right), T_{2}\right) \wedge\right. \\
& \left.T \preceq T_{1}+1\right) \\
& \rightarrow \mathbf{P} \text { delete }(r, \text { Position }(x, y),[T, n o w])
\end{aligned}
$$


with $T_{1}+1$ being an operation which adds one minute to time $T_{1}$ (Cuppens and Saurel, 1997).

Then the third step consists in giving the integrity evolution policy. We first define a predicate max_hold $\left(p,\left[T_{1}, T_{2}\right]\right.$ ) as follows (see also Sripada (Sripada, 1993)):

- $\forall T_{1}, \forall T_{2}$, max $h$ hold $\left(p,\left[T_{1}, T_{2}\right]\right) \leftrightarrow$

$$
\begin{array}{ll} 
& \operatorname{hold}\left(p,\left[T_{1}, T_{2}\right]\right) \\
\wedge & \neg \exists T, T \prec T_{1} \wedge \operatorname{hold}\left(p,\left[T, T_{2}\right]\right) \\
\wedge & \neg \exists T, T_{2} \prec T \wedge \operatorname{hold}\left(p,\left[T_{1}, T\right]\right)
\end{array}
$$

that is: we have max hold $\left(p,\left[T_{1}, T_{2}\right]\right)$ if and only if $p$ is valid on interval $\left[T_{1}, T_{2}\right]$ and there is no interval $I$ containing $\left[T_{1}, T_{2}\right]$ such as $p$ is valid on $I$.

The integrity evolution is then defined as follows:

- $\forall T, \forall T_{1}, \forall x, \forall y$

Reference_date $(T) \wedge$

hold_at (believe $\left(H I\right.$, max hold $\left(\right.$ Position $(x, y),\left[T_{1}\right.$, now $\left.\left.\left.]\right)\right), T\right) \wedge$

$T_{1}+1 \preceq T$

$\rightarrow \mathbf{O}$ revise_integrity $\left(\operatorname{Position}(x, y),\left[T_{1}+1\right.\right.$, now $\left.], M I\right)$

that is: if the database believes at time $T$ that the position of the ship has been highly reliable since time $T_{1}$, and if the reference date is more than one minute after $T_{1}$, then the level of integrity of the position of the ship has to be revised to the medium level of integrity.

$\forall T, \forall T_{1}, \forall x, \forall y$

Date_reference $(T) \wedge$

hold_at (believe $\left(M I\right.$, max hold $\left(\right.$ Position $(x, y),\left[T_{1}\right.$, now $\left.\left.\left.]\right)\right), T\right) \wedge$ $T_{1}+2 \preceq T$

$\rightarrow \mathrm{O}$ revise_integrity $\left(\operatorname{Position}(x, y),\left[T_{1}+2\right.\right.$, now $\left.], L I\right)$

(similar to the previous one: the level of integrity of the position has to be revised to a low level of integrity after two minutes).

Let us see how this integrity policy applies to a practical example. Let us assume that at 12:00, the satellite $s$ provides the data: "the position of the ship $s h$ is pos 1 at 12:00". 
- hold_at(insert(s, Position(sh, pos1), [12:00, now]), 12:00)

Since $s$ is allowed to insert this data, the database includes the following data :

- hold(believe(HI, Position(sh, pos1), [12:00, now]), [12:00, now])

According to the policy, the radar is not allowed to update this data between 12:00 and 12:01. So, let us assume that at 12:01, $r$ provides data corresponding to: the position of $s h$ is pos2 at time 12:01. As an update corresponds to a deletion followed by an insertion, we have :

- hold_at(delete(r, Position(sh, pos2), [12:01, now]), 12:01)

- hold_at(insert(r, Position(sh,pos2), [12:01, now]), 12:01)

Since these operations are allowed for the radar $r$, the database then contains the facts :

- hold(believe(HI, Position(sh,pos1), [12:00, now]), [12:00, 12:01[)

- hold(believe(HI, Position(sh,pos1), [12:00, 12:01[), [12:01, now])

- hold(believe(LI, Position(sh,pos2), [12:01, now]), [12:01, now])

and so on.

Now, let us assume that $r$ does not provide any information between $12: 00$ and $12: 05$, then according to the integrity evolution policy the state of the database at time 12:05 will be:

- hold(believe(HI, Position(sh, pos1), [12:00, now]), [12:00, 12:01[)

- hold(believe(HI, Position(sh, pos1), [12:00, 12:01[), [12:01, now])

- hold(believe(MI,Position(sh,pos1),[12:01, now]), [12:01, 12:03[)

- hold(believe(MI, Position(sh,pos1), [12:01, 12:03[), [12:03, now])

- hold(believe(LI, Position(sh, pos 1$),[12: 03$, now $]),[12: 03$, now $])$

\subsection{EXAmple 2: In a COMPany}

We introduce the following predicates: 
- Employee $(x): x$ is an employee.

- Secretary $(x): x$ is a secretary.

- Temporary $(x): x$ est a temporary employee.

- In_vacation $(x): x$ is in vacation.

- $\operatorname{Val} S a l(x): x$ is a salary value.

- Salary $(x, y): x$ 's salary is $y$.

Here are integrity constraints:

- $\forall x, \operatorname{Secretary}(x) \rightarrow$ Employee $(x)$

- $\forall x, \forall y, \operatorname{Salary}(x, y) \rightarrow E m p l o y e e(x) \wedge \operatorname{Val}$ SSal $(y)$

- $\forall x, \forall y, \forall y^{\prime}, \operatorname{Salary}(x, y) \wedge \operatorname{Salary}\left(x, y^{\prime}\right) \rightarrow y=y^{\prime}$

Now, we consider we have three agents: two secretaries $s 1$ and $s 2$, and a temporary employee $s 3$. We first specify their respective reliability:

- $\forall x, \forall y, \forall T, E$ Employee $(x) \wedge V a l \_S a l(y) \wedge \operatorname{Date}(T)$

$\rightarrow$ safer_than $(s 1$, hold (Salary $(x, y),[T$, now $]), H I)$

( $s 1$ is a high reliable source for salaries)

- $\forall x, \forall T_{1}, \forall T_{2}, E m p l o y e e(x) \wedge \operatorname{Date}\left(T_{1}\right) \wedge \operatorname{Date}\left(T_{2}\right)$

$\rightarrow$ safer_than $\left(s 2\right.$, hold(In_vacation $\left.\left.(x),\left[T_{1}, T_{2}\right]\right), H I\right)$

( $s 2$ is highly reliable for vacations. $T_{2}$ must be a date, not the special value now)

- $\forall x, \forall y, \forall T, E m p l o y e e(x) \wedge V a l \_S a l(y) \wedge \operatorname{Date}(T)$

$\rightarrow$ safer_than $(s 3$, hold $(\operatorname{Salary}(x, y),[T$, now $]), M I)$

( $s 3$ is a medium reliable source for salaries)

- $\forall x, \forall T_{1}, \forall T_{2}, E m p l o y e e(x) \wedge \operatorname{Date}\left(T_{1}\right) \wedge \operatorname{Date}\left(T_{2}\right)$

$\rightarrow$ safer_than $\left(s 3\right.$, hold $\left(\right.$ In_vacation $\left.\left.(x),\left[T_{1}, T_{2}\right]\right), H I\right)$

( $s 3$ is highly reliable for vacations)

The second step consists in defining the updating policy as follows : 
- $\forall x, \forall y, \forall T, E m p l o y e e(x) \wedge \operatorname{Val} \operatorname{Sal}(y) \wedge \operatorname{Date}(T)$

$\rightarrow \mathbf{P}$ insert $(s 1, \operatorname{Salary}(x, y),[T$, now $])$

- $\forall x, \forall y, \forall T, E m p l o y e e(x) \wedge \operatorname{Val} \_S a l(y) \wedge \operatorname{Date}(T)$

$\rightarrow \mathbf{P}$ delete $(s 1, \operatorname{Salary}(x, y),[T$, now $])$

( $s 1$ is allowed to update (insert then delete) salaries of employees)

- $\forall x, \forall T_{1}, \forall T_{2}, E$ Employee $(x) \wedge \operatorname{Date}\left(T_{1}\right) \wedge \operatorname{Date}\left(T_{2}\right)$

$\rightarrow \mathbf{P}$ insert $\left(s 2\right.$, In_vacation $\left.(x),\left[T_{1}, T_{2}\right]\right)$

- $\forall x, \forall T_{1}, \forall T_{2}, E$ Employee $(x) \wedge \operatorname{Date}\left(T_{1}\right) \wedge \operatorname{Date}\left(T_{2}\right)$

$\rightarrow \mathbf{P}$ delete $\left(s 2\right.$, In_vacation $\left.(x),\left[T_{1}, T_{2}\right]\right)$

( $s 2$ is allowed to update data corresponding to the vacation of employees).

- $\forall x, \forall y, \forall T$,

Employee $(x) \wedge \operatorname{Val} \_\operatorname{Sal}(y) \wedge \operatorname{Date}(T) \wedge$

In_vacation $(s 1)$

$\rightarrow \mathbf{P}$ insert $(s 3, \operatorname{Salary}(x, y),[T$, now $])$

- $\forall x, \forall y, \forall T$,

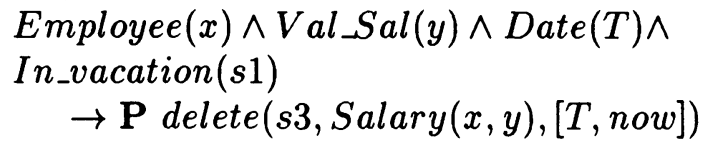

(during $s 1$ 's vacation, $s 3$ is allowed to update salaries of employees).

- $\forall x, \forall T_{1}, \forall T_{2}$,

Employee $(x) \wedge \operatorname{Date}\left(T_{1}\right) \wedge \operatorname{Date}\left(T_{2}\right) \wedge$

In_vacation $(s 2)$

$\rightarrow \mathbf{P}$ insert $\left(s 3, \operatorname{In\_ vacation}(x),\left[T_{1}, T_{2}\right]\right)$

- $\forall x, \forall T_{1}, \forall T_{2}$,

$\operatorname{Employee}(x) \wedge \operatorname{Date}\left(T_{1}\right) \wedge \operatorname{Date}\left(T_{2}\right) \wedge$

In_vacation $(s 2)$

$\rightarrow \mathbf{P}$ delete $\left(s 3\right.$, In_vacation $\left.(x),\left[T_{1}, T_{2}\right]\right)$

(if $s 2$ is in vacation, then $s 3$ is allowed to update data corresponding to the vacation of employees). 
Finally, we assume that there is no rule to specify how data integrity changes in time. Therefore, the integrity policy is now completely defined.

\section{Conclusion}

In this paper, we have presented a formal model to specify integrity policies for database management systems. A major difference with previous proposals is that this model includes an explicit representation of time. This allows us to:

1. Manage historical data. It is possible to update a highly reliable data by a lower one; the highly reliable data is not deleted from the database but recorded in the history. This represents a major difference with Biba and corresponds to real practical requirements.

2. Label data with transaction time. This makes it possible to revise the integrity level of "old" data.

3. Model integrity policies where rights may change in time (as in (Bertino et al., 1996)).

We can also make the following comments when we try to compare our model with Clark-Wilson (Clark and Wilson, 1987). Clark-Wilson's model is based on two basic concepts:

- Separation of duty. This corresponds to the requirement that several different agents may be necessary to perform a given task. This requirement is not explicitly included in our model but we guess that using the concepts of permission and prohibition, it would be easily possible to specify this kind of notion in our model. See also (Sandhu, 1988) for some practical ideas to deal with separation of duty in database management systems.

- Well formed transaction. This corresponds to the requirement that any transaction should transform a valid system state into another valid system state. As mentioned before, this essentially corresponds to the notion of integrity constraints which is implemented in most database management systems. 
In Clark-Wilson, rights are represented by triples (agent, procedure, set of data items) which specifies that a given agent is allowed to access a given set of data by using a given procedure. Our model refines ClarkWilson's triples by including the possibility to specify rights that may change in time.

In (Sandhu and Jajodia, 1991), Sandhu and Jajodia also investigate basic integrity principles and suggest mechanisms to implement them in the case of a database management system. Their conclusion is that classical database management systems already include several mechanisms (in particular integrity constraint management) which may be directly used to implement these integrity principles. They also mentioned that delegation of authority is an important requirement when specifying integrity policies. This requirement is not represented in our model (nor it is in Clark-Wilson's one). This represents further refinement that remains to be done.

Finally, let us mention that our approach has been used to model the integrity policy of a police application which manage a database of criminal cases. In particular, the fact that the integrity level of data may change in time corresponds to a practical requirement in this application. It is also necessary to accept low reliable clues even though there are already higher reliable clues in the database; and of course, there are various classes of agents and their rights generally change in time. Therefore, this application fits well with the various concepts which are included in our integrity policy model.

\section{Acknowledgement}

This work was supported by DSP/STTC $94002-073$ contract.

\section{References}

Bell, D. and L. LaPadula: 1975, 'Secure Computer Systems: Unified Exposition and Multics Interpretation'. Technical Report ESD-TR-75-306, MTR-2997, MITRE, Bedford, Mass.

Bertino, E., C. Bettini, E. Ferrari, and P. Samarati: 1996, 'A Temporal Access Control Mechanism for Database Systems'. IEEE Transactions on Knowledge and Data Engineering 8(1).

Biba, K.: 1976, 'Integrity Considerations for Computer Systems'. Technical Report ESD-TR-76-372, MITRE, Bedford, Mass. 
Chellas, B. F.: 1988, Modal Logic: An Introduction. Cambridge University Press.

Clark, D. and D. Wilson: 1987, 'A Comparison of Commercial and Military Computer Security Policies'. In: IEEE Symposium on Security and Privacy. Oakland.

Clark, D. and W. Wilson: 1989, 'Evolution of a Model for Computer Integrity'. In: Z. Ruthberg and W. Polk (eds.): Report of the Invitational Workshop on Data Integrity. Gaithersburg.

Clifford, J., C. Dyreson, T. Isakowitz, C. Jensen, and R. Snodgrass: 1997, 'On the Semantics of "Now" in Databases'. ACM Transactions on Database Systems 22(2), 171-214.

Cuppens, F. and C. Saurel: 1997, 'Outil de classification et de déclassification de documents multiniveaux'. Rapport 3/3564.00/DERI, Centre d'études et de recherches de Toulouse.

Cuppens, F. and C. Saurel: 1998, 'Dimension temporelle dans les systèmes sécurisés : évolution de l'intégrité des données dans le temps'. Rapport final 2/3700.00/DTIM, Centre d'études et de recherches de Toulouse.

Meyer, J.-J. and R. Wieringa: 1991, 'Deontic Logic: A Concise Overview'. In: Proc. of the First International Workshop on Deontic Logic in Computer Science. Amsterdam, The Netherlands. Tutorial.

Sandhu, R.: 1988, 'Transaction Control Expressions for Separation of Duties'. In: Proc. 4th Aerospace Computer Security Applications Conference. Orlando, Florida.

Sandhu, R. and S. Jajodia: 1991, 'Integrity Principles and Mechanisms in Database Management Systems'. Computer \& Security 10, 413-427.

Snodgrass, R. and I. Ahn: 1985, 'A taxonomy of time in databases'. In: Proceedings of the ACM SIGMOD Conference on Management of Data. Austin, TX.

Sripada, S. M.: 1993, 'A Metalogic Programming Approach to Reasoning about Time in Knowledge bases'. In: Proceedings of the 13th International Joint Conference on Artificial Intelligence. 